\title{
Non-Invasive and Non-Destructive Determination of Corneal and Scleral Biomechanics Using Vibrational Optical Coherence Tomography: Preliminary Observations
}

\author{
Frederick H. Silver ${ }^{1 *}$, Ruchit G. Shah ${ }^{2}$, Dominick Benedetto ${ }^{3}$ \\ ${ }^{1}$ Department of Pathology and Laboratory Medicine, Robert Wood Johnson Medical School, Rutgers, The State University of New \\ Jersey, Piscataway, NJ, USA \\ ${ }^{2}$ Graduate Program in Biomedical Engineering, Rutgers, The State University of New Jersey, Piscataway, NJ, USA \\ ${ }^{3}$ Morristown Ophthalmology Associates, Morristown, NJ, USA \\ Email: *silverfr@rutgers.edu, ^fhsilver@hotmail.com
}

How to cite this paper: Silver, F.H., Shah, R.G. and Benedetto, D. (2018) Non-Invasive and Non-Destructive Determination of Corneal and Scleral Biomechanics Using Vibrational Optical Coherence Tomography: Preliminary Observations. Materials Sciences and Applications, 9, 657-669.

https://doi.org/10.4236/msa.2018.97047

Received: April 24, 2018

Accepted: June 26, 2018

Published: June 29, 2018

Copyright ( $) 2018$ by authors and Scientific Research Publishing Inc. This work is licensed under the Creative Commons Attribution International License (CC BY 4.0).

http://creativecommons.org/licenses/by/4.0/ (c) (i) Open Access

\begin{abstract}
Experimental measurements made in this study on human and porcine eyes suggest that the resonant frequency for both cornea and sclera varies from 130 to $150 \mathrm{~Hz}$ and increases slightly with increasing intraocular pressure. The values of the moduli calculated using the experimental values of the thickness are close to $2 \mathrm{MPa}$. Similar values of the modulus for cornea and sclera suggest that there is very little stress concentration at the cornea-scleral junction and that any stress concentration that occurs probably resides at the scleral attachment laterally and posteriorly. These moduli are close to those measured in vivo on human skin suggesting that the mechanism of tensile deformation of skin, cornea and sclera are similar. Our results suggest that the modulus of cornea and sclera can be measured non-invasively and non-destructively using vibrational OCT. Results of these studies will assist clinicians to better understand the influence of biomechanics on the outcome of corneal refractive surgery as well as the pathogenesis of eye disorders such as glaucoma, myopia and keratoconus.
\end{abstract}

\section{Keywords \\ Collagen, Cornea, Sclera, Skin, Biomechanics, Vibrational OCT, Optical Coherence Tomography (OCT), Modulus, Resonant Frequency, Mechanical Properties, Myopia, Keratoconus, Glaucoma}




\section{Introduction}

The cornea and its tear film form the anterior superficial structure of the eye. The cornea functions both as a clear optical lens, focusing light on the retina and a protective structure for the eye's inner elements. It is an aspheric, prolate (steeper centrally than peripherally), curved structure and the most significant refractive element of the eye providing 45 diopters of the total 65 diopters of refractive power for an average sized eye.

Corneal clarity is maintained by the uniform spacing of its stromal collagen fibrils [1] which comprise $80 \%$ of the cornea's structure and the majority of its strength [2]. The biomechanics of the cornea has been studied extensively in an attempt to understand and predict the response of the cornea to surgical and external trauma as well as its response to common systemic and corneal degenerative diseases [3] [4] [5] [6].

Corneal incisions, laser reshaping procedures and systemic and corneal degenerative diseases can weaken corneal structure. In some cases, these influences can lead to unpredictable changes in corneal shape and clarity. Collagen cross linking with UV activated riboflavin has the capability to stiffen and strengthen the stroma [7].

Corneal biomechanics has been evaluated by in vitro studies of tensile properties of corneal samples, pressure displacement studies of inflated whole human ocular samples, and in vivo measurements of corneal displacement by air puff indentation [3]-[11]. The need for a standard method to calibrate ocular biomechanical measurements makes it difficult to interpret many of the studies reported in the literature. For example, the reported moduli of the cornea vary from 0.250 $\mathrm{MPa}$ [3] to over $35 \mathrm{MPa}$ [9] [10]. This large variation in measurement may be due to: 1) strain dependence of the mechanical behavior, 2) strain-rate dependence 3) assumption that the tissue deforms at constant volume, and 4) difficulties in measuring the tangent to the stress-strain curve required to calculate the modulus [12].

The corneal stromal is composed of layers of collagen fibrils that are oriented at different angles depending on the anatomic location. The stroma is reported to be composed of $8 \%$ water, $15 \%$ collagen and $7 \%$ non-collagenous proteins and provides the tensile resistance (stiffness also termed modulus) to deformation [3] [4] [5].

A variety of methods have been used to evaluate the mechanical properties of extracellular matrix (ECM) over the last 30 years including uniaxial and biaxial tensile testing, indentation and rotational tests, ultrasound elastography (UE), optical coherence tomography (OCT), optical coherence elastography (OCE), and OCT combined with vibrational analysis [12] [13] [14] [15]. Some of these techniques assume the following materials properties: that the material is linear elastic, Poisson's ratio is close to 0.5 (stretching occurs at constant volume) and that viscoelasticity does not dramatically affect the resulting properties [12]. In addition, ECMs are non-linear materials that have an upward curvature to the 
stress-strain curve. This makes determination of the stiffness (tangent to the stress-strain curve) and other mechanical properties very difficult to quantify since the tangent to the stress-strain curve is constantly changing [12] [13] [14] [15].

We recently reported the use of OCT and vibrational analysis to image and evaluate the mechanical properties of a number of ECMs including human skin, decellularized human dermis, pig skin, human scar, bovine cartilage, and bovine subchondral bone [12] [16] [17]. The correlation between modulus measurements made on human dermis using standard tensile testing in vitro and vibrational OCT in vitro suggests that the two methods give similar values of the modulus [16] [17]. Beyond these calibration studies, vibrational OCT has been used in vivo to image and report the differences in mechanical properties of human skin and scar tissues [17]. In this paper, we report images and preliminary biomechanical observations of the elastic modulus of porcine and human cornea and sclera using vibrational OCT measurements on whole eyes. The calculated values of modulus are compared to values reported for other ECMs composed of fibrous collagens.

\section{Methods}

\subsection{Sample Preparation}

Human decellularized dermis was obtained from allograft tissue as described previously and was tested after immersion in phosphate buffer solution as described elsewhere [16] [17]. All samples were tested wet after soaking in phosphate buffer solution at $\mathrm{pH} 7.4$ for at least 30 minutes. Processing and testing steps were conducted at $22^{\circ} \mathrm{C}$.

Human and porcine whole eyes were tested in vitro to demonstrate the use of the OCT and vibrational techniques to measure the modulus of ocular tissues. The porcine eyes were obtained from Spear Products (Coopersburg, $\mathrm{Pa}$ ) and the human eyes were obtained from Eversight Eye Bank (Chicago, IL).

\subsection{Imaging}

OCT images of cornea and sclera were made using a Lumedica OQ labscope (Lumedica, Inc., Durham, NC) operating in the scanning mode. Sample thicknesses for cornea and sclera were measured from OCT images using the Lumedica device as well as the laboratory OCT [16] [17]. OCT images of the cornea and sclera are shown in Figure 1.

\subsection{Mechanical Testing}

Incremental Stress-Strain Tensile Measurements in Vitro.

Calibration samples of decellularized human dermis were tested in uniaxial tension at $22^{\circ} \mathrm{C}$ by adding a strain increment and then measuring the load before an additional strain step was added as described previously [16] [17]. Axial deformations were applied through adjustment of a graduated translation stage. 

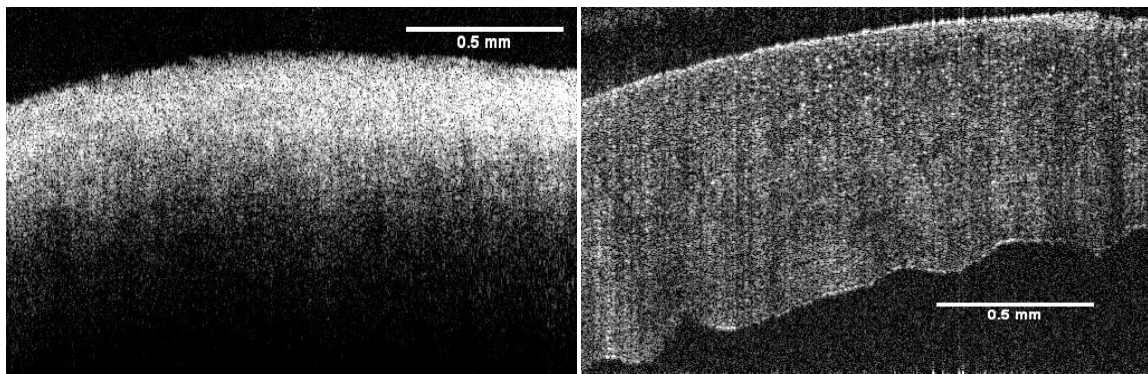

Figure 1. Typical images of the cornea (right) and sclera(left) from a human eye bank eye obtained using a Lumedica OQ Labscope at low intraocular pressure $(7 \mathrm{~mm} \mathrm{Hg})$.

The resulting axial force was measured by the force gage and recorded for subsequent calculations. Stress values were calculated from the force divided by the cross-sectional area. Strains were calculated by dividing the change in length by the original length based on the movement of the translational stage after each strain increment was added. The tensile modulus was calculated from a tangent drawn to the stress-strain curve at the strain increment used as described previously [16] [17].

\subsection{Calibration Studies}

A variety of samples made from silicone rubber, decellularized human dermis, pig skin, and chemically modified decellularized dermis were tested in uniaxial tension and using vibrational analysis to establish a calibration curve between the moduli calculated from tensile measurements and those derived from vibrational measurements in vitro. These results have been published elsewhere [16] [17].

The relationship between the modulus measured using vibrational and tensile measurements was reported to be approximately linear and the equation of the line was found to be:

$$
E v=1.026 E t+0.0046
$$

where, $E_{V}$ and $E t$ are the moduli measured using vibrational and tensile measurements, respectively and are in MPas. The correlation coefficient between these moduli is 0.984 as previously reported [16] [17]. The relationship between tensile and vibrational moduli was approximated using Equation (1). The material behavior was reported to be reversible for strains less than about $14 \%$ and for at least three cycles of tensile testing [16] [17]. At the resonant frequency, the modulus measured is within $3 \%$ to $4 \%$ of being perfectly elastic [16] [17].

The viscous component was estimated from the driving frequency peak, by dividing the change in frequency at the half height of the peak (i.e. $3 \mathrm{db}$ down from maximum peak in power spectrum) by the driving frequency. This method is known as the half-height bandwidth method discussed by Paul Macioce (www.roush.com/wp-content/uploads/2015/09/Insight.pdf) [18].

\subsection{Vibrational OCT Experiments in Vitro}

Transverse forces were applied to the sample by positioning an acoustic loud- 
speaker (IntervoxS225RA-40) beneath the sample. A function generator (Agilent) was used to drive the speaker with sinusoidal waveforms at varying amplitudes and frequencies as described previously [16] [17].

Transverse sample displacement was measured by spectral-domain optical coherence tomography (SD-OCT), a non-contact, interferometric technique as discussed previously [16] [17]. The SD-OCT system uses a fiber-coupled superluminescent diode light source with $1325 \mathrm{~nm}$ center wavelength and $100 \mathrm{~nm}$ bandwidth (full-width at half maximum) [16] [17].

The resonant frequency of each sample was initially estimated at a single point by measuring the transverse displacement resulting from sinusoidal driving frequencies ranging from $50 \mathrm{~Hz}$ to $1000 \mathrm{~Hz}$, in steps of $50 \mathrm{~Hz}$ as described previously [16] [17]. Once the region where the maximum frequency was identified, smaller steps of $10 \mathrm{~Hz}$ were used to more accurately identify the peak frequency and the actual resonant frequency, $f_{n}$.

$$
E=m\left(2 \pi f_{n}\right)^{2}\left(\frac{L}{A}\right)
$$

The modulus, E, from in vitro vibrational studies was determined using Equation (2) where $m, L$ and $A$ are the sample mass, length and cross-sectional area. The resonant frequency was determined after correction for the resonant frequencies exhibited by the speaker and any interference due to line fluctuations.

\subsection{In Vitro Determination of the Resonant Frequency of Cornea and Sclera}

In vitro studies of the mechanical properties of cornea and sclera were conducted by fixing the whole eye in a Styrofoam block using needles and then vibrating the eye from beneath the block using the sinusoidal sound waves. The block displacements as a function of frequency were measured without the eye in place and were compared to the displacements measured in the presence of the eye. The resonant frequency of the cornea and sclera were converted into a modulus using Figure 2, a calibration curve of modulus times sample thickness versus resonant frequency for decellularized human dermis, porcine tissues, bovine cartilage, and human skin. The sample thickness was determined from OCT images.

Corneas were inflated by injecting normal saline into the globe using a needle and syringe. Human eyes were inflated to intraocular pressures of $7 \mathrm{~mm} \mathrm{Hg}$ (low pressure) and $60 \mathrm{~mm} \mathrm{Hg}$ (high pressure); the pressure was measured using a Schiotz Tonometer (Tiger Medical, Irvington, NJ). Eyes were mounted in a support structure carved out of a block of Styrofoam that was clamped in place using a compressive force applied by mechanical grips at either end of the block. The whole eye was held in place by inserting three or more needles through the scleral insertion of the ocular rectus muscles anchoring them to the Styrofoam. The resonant frequency of the cornea and sclera were converted into values of the modulus using Figure 2 and the experimentally measured sample thickness determined from OCT images. 


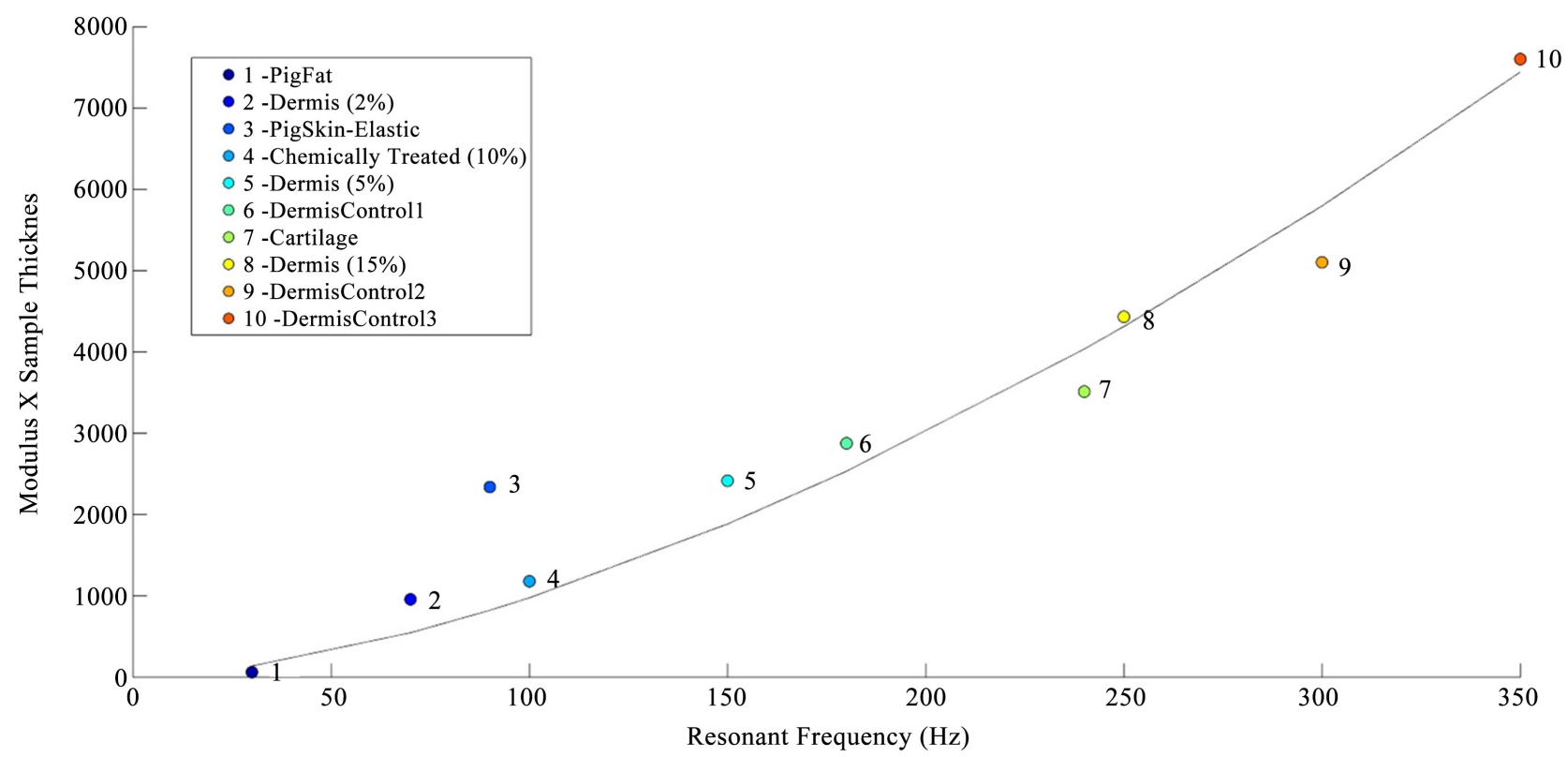

Figure 2. Calibration curve showing the relationship between the product of the modulus (E) times the tissue thickness versus resonant frequency measured using vibrational OCT for different tissues. The calibration curve includes points from pig fat and elastic tissue, human decellularized dermis at different strains, chemically modified decellularized dermis, and bovine femoral cartilage.

\section{Results}

Stress-strain curves for inflated eyes obtained from the literature [18] were compared to those of decellularized human dermis previously published [16] [17] as shown in Figure 3. The stress-strain curves for decellularized human dermis are similar in shape to the curves for porcine and human eyes consistent with other tissues containing fibrous collagen networks [15]. These curves show increased slopes with increasing strains. The tensile stress-strain curves of decellularized human dermis and inflated eyes are composed of low and high modulus regions consistent with previous reports on mechanical measurements on human dermis and skin [15] [16] [17]. The difference in the length of the low modulus region is related to the strain required to recruit the collagen fibrils and fibers along the tensile direction [15]. The similarity in the shape of the stress-strain curves between human and porcine eyes and decellularized dermis indicate that the stress-strain behavior has a similar mechanism in these tissues, e.g., at low strains the collagen fibrils and fibers are only partially recruited in the loading direction and become fully recruited at high strains increasing the value of the modulus.

Vibrational OCT was used to measure the mechanical properties of porcine and human corneas and scleras in vitro. The results of previously published data on decellularized human dermis, human skin, pig skin, human scar, and bovine cartilage were used to calibrate and interpret the in vitro studies. The resonant frequencies of human cornea and sclera were measured from vibrational OCT studies at both low $(7 \mathrm{~mm} \mathrm{Hg})$ and high $(50 \mathrm{~mm} \mathrm{Hg})$ intraocular pressures as shown in Figure 4. Resonant frequencies were obtained by determining the fre- 
quency at which the maximum displacement was observed based on measurements at a single point about $14 \mu \mathrm{m}$ in diameter. The resonant frequencies and calculated moduli for porcine cornea and sclera and human cornea and sclera from whole eye measurements are listed in Table 1 . The calculated values of the modulus for cornea and sclera were determined using Figure 2 and are about 2 MPas (Table 1) similar to those reported for decellularized human dermis and human skin [16] [17]. Note the viscous loss reported for cornea and sclera at the resonant frequency is between $2 \%$ and $3 \%$ (Table 1 ).

Table 1. Resonant frequency and calculated modulus for central cornea, corneal-scleral junction, and sclera at low $(7 \mathrm{~mm} \mathrm{Hg})$ and high $(50 \mathrm{~mm} \mathrm{Hg})$ intraocular pressures. The viscous response is presented as a percent of the elastic response.

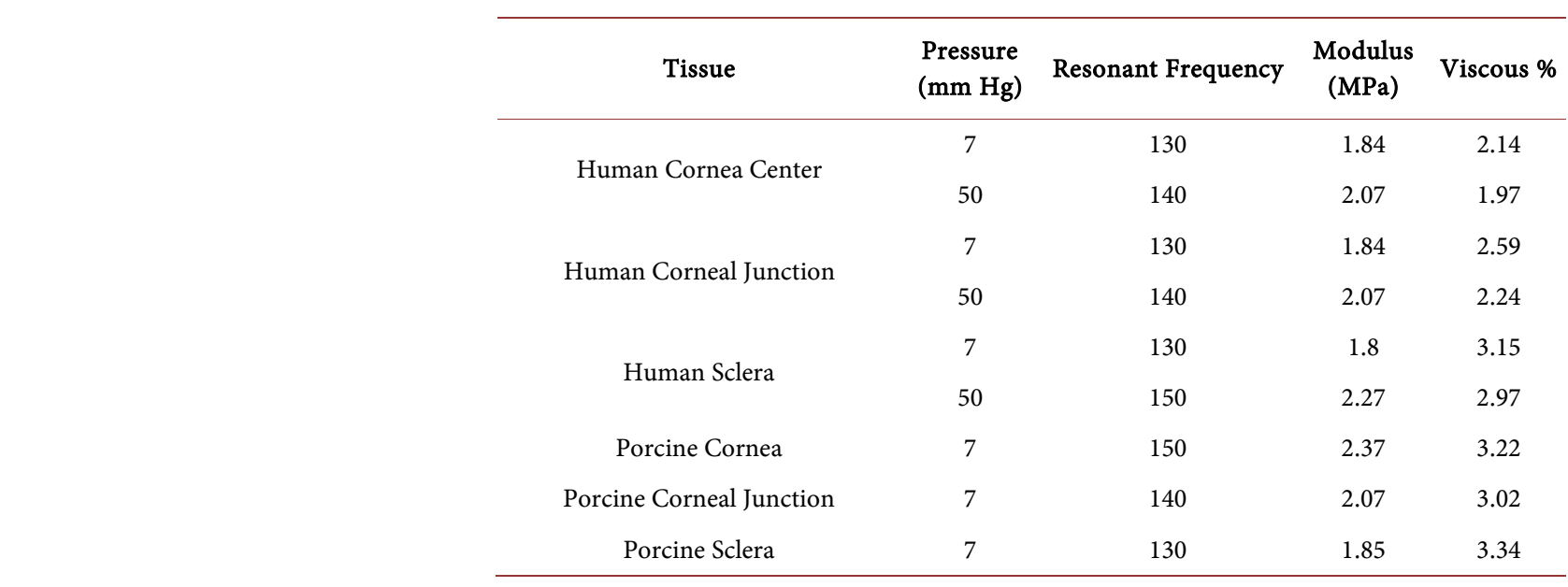

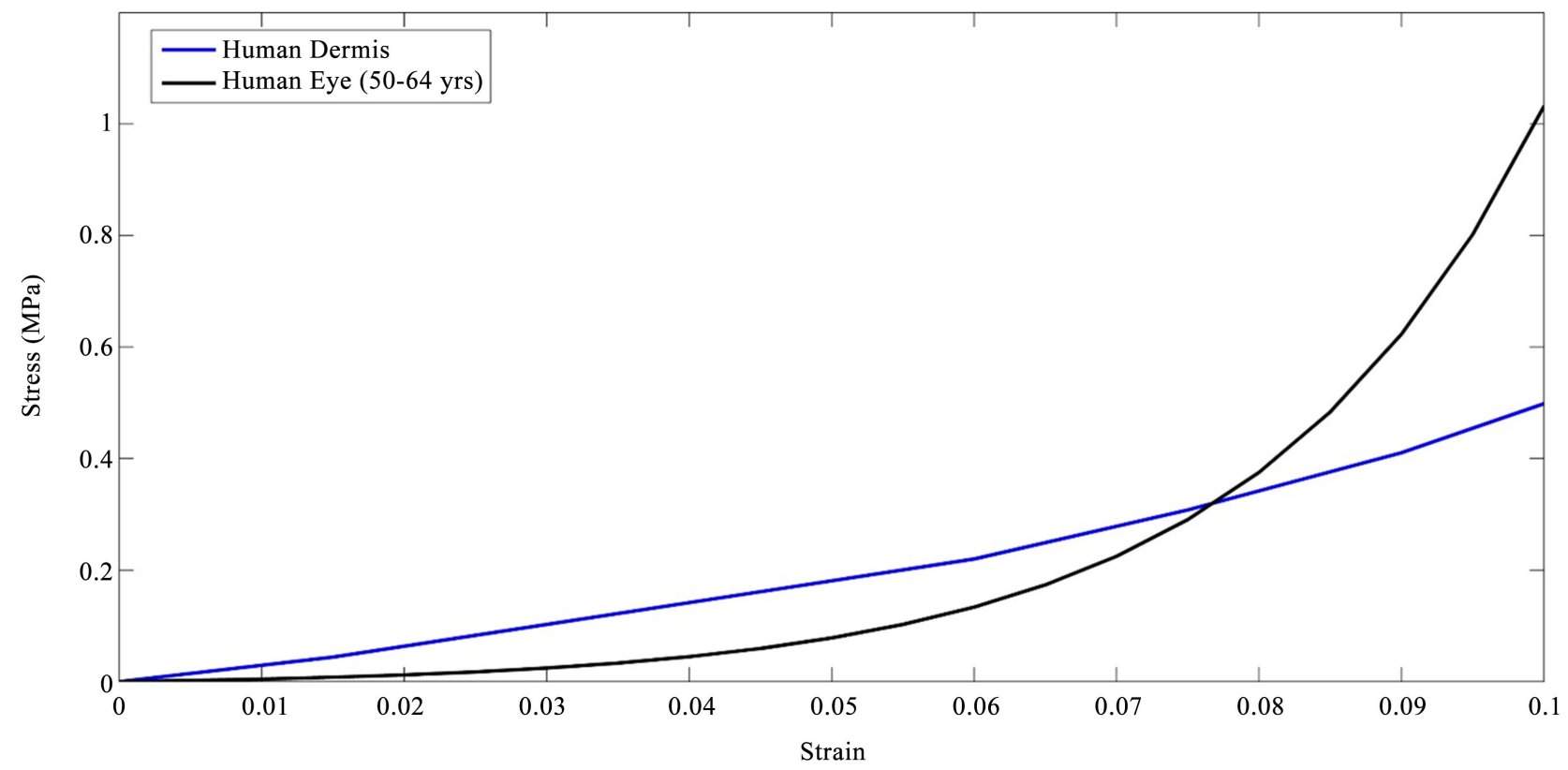

Figure 3. Tensile stress-strain curve for decellularized dermis obtained from tensile stress-strain measurements [16] [20] and human eyes from inflation tests [19]. Note the similarity between the shapes of the stress-strain curve for decellularized dermis composed largely of type I collagen and that of whole eyes. The upward curvature involves increased recruitment of collagen fibers with the loading direction with increasing strain and leads to the increased slope of the stress-strain curve. 


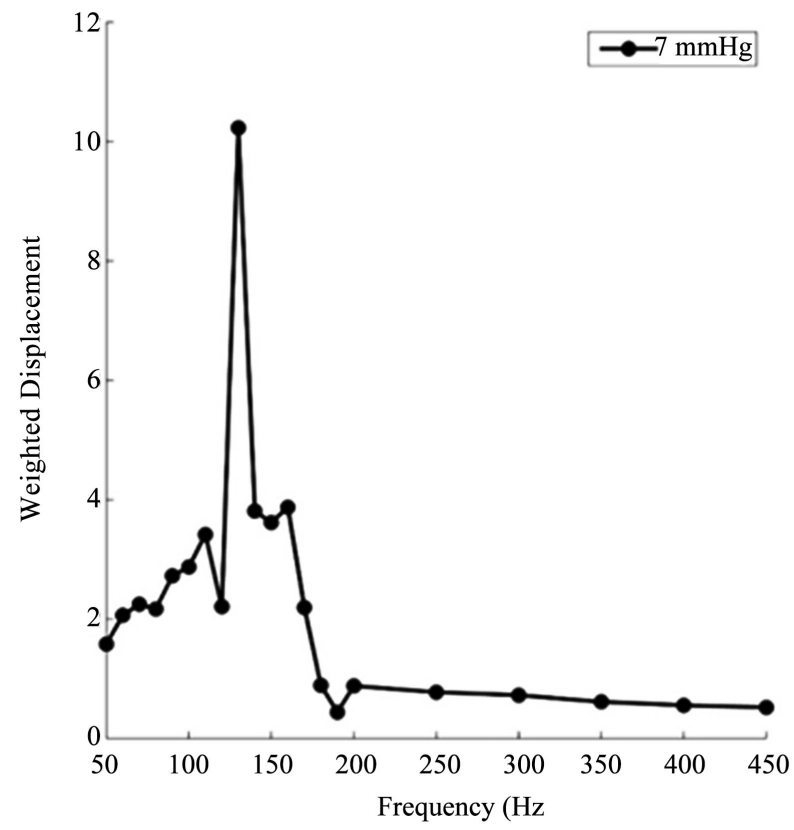

(a)

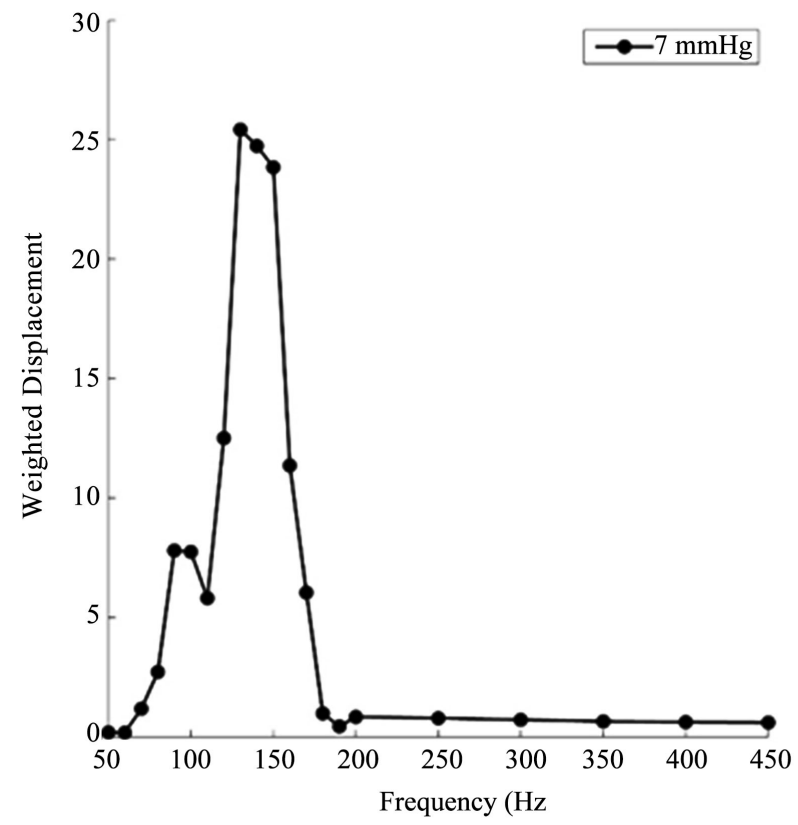

(c)

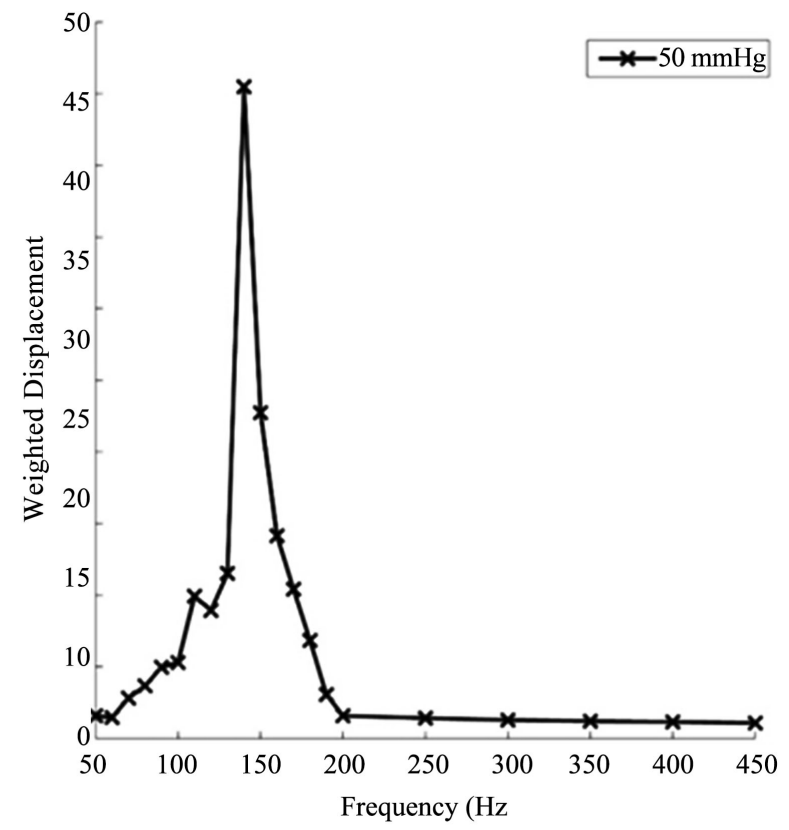

(b)

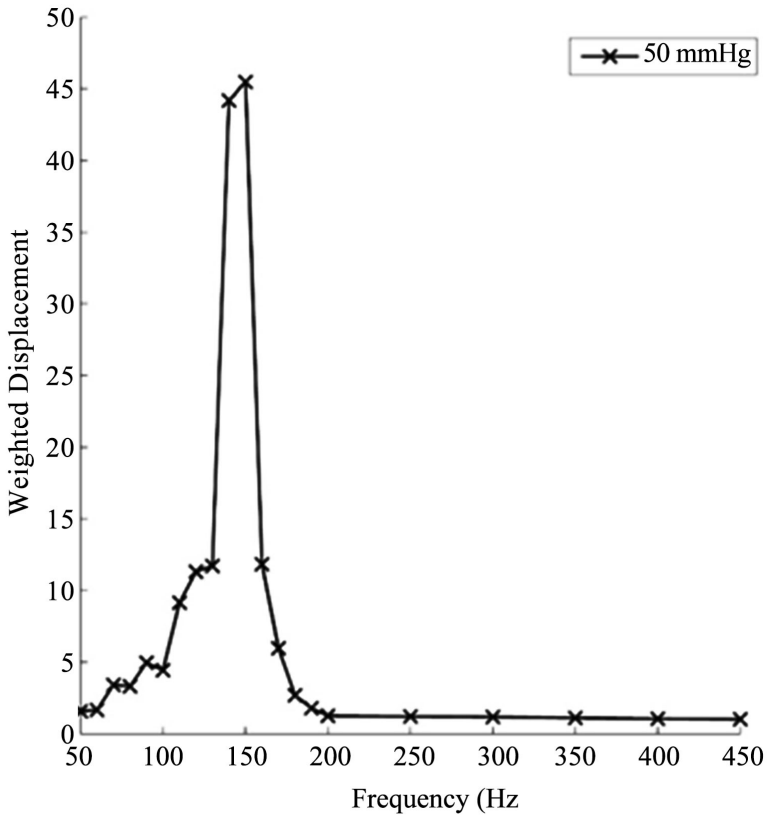

(d)

Figure 4. Weighted displacement versus frequency for human cornea (a) (b) and sclera (c), (d) at low (7 mm $\mathrm{Hg}$ ) and high (50 $\mathrm{mm} \mathrm{Hg}$ ) pressures. The resonant frequency is determined as the frequency at which the maximum displacement occurs. Note peaks from the Styrofoam blocks and line noise were removed. The resonant frequency changes are small with increased pressure. The increased moduli at higher pressures are listed in Table 1.

\section{Discussion}

The limited ability of researchers and clinicians to correlate changes in OCT images of cornea and sclera with the biomechanics of the ECM underscores the need to develop new non-invasive methods to understand and diagnose the nature of common eye disorders and surgical interventions [6]. While the me- 
chanical properties of ECMs are complex, much progress has been made in understanding the strain-rate dependence, non-linearity and compressibility of these tissues [15] [16] [17]. By calculating the modulus at the resonant frequency it is possible to eliminate the viscous contribution to the mechanical properties [20]. By pulsing tissues with a series of sinusoidal sound waves as a function of the frequency, the viscous contribution can be shown to almost disappear at the resonant frequency [20]. The viscous contribution of skin has been shown to as high as $25 \%$ at low strains and strain-rates [15] and as low as $3 \%$ to $4 \%$ at high strains and strain-rates [20]. However, measurements made at the resonant frequency simplify the analysis since they are independent of strain-rate.

The correlation between modulus measurements on decellularized human dermis made using standard tensile testing in vitro and vibrational OCT suggest that measurements made using vibrational OCT give results that are consistent with tensile testing, a "gold standard method" for measuring mechanical properties of ECMs [15] [16] [17]. Without comparison to a standard technique, moduli measurements made with new methods such as vibrational OCT cannot be validated [12].

Tensile incremental and constant rate-of-strain measurements made on tissues have been the gold standard for determination of the mechanical properties of tissues for decades [13] [14] [15]. Many techniques require the assumption that the tissue density is near 1.0 and that Poisson's ratio is 0.5 (stretching without a change in volume). The later has been shown to vary between 0.38 and 0.75 for decellularized dermis [21]. The assumption that Poisson's ratio is 0.5 will lead to errors in modulus calculations. However, by measuring the modulus at the resonant frequency using vibrational analysis and OCT and using Equations (1) and (2), the elastic modulus can be calculated for ECMs without the need to make any assumptions.

Experimental measurements made on human and porcine eyes suggest that the resonant frequency for human and porcine cornea and sclera vary from 130 to $150 \mathrm{~Hz}$ and increase slightly with increasing pressure. The values of the moduli calculated from Figure 3 using the experimental values of the thickness are about $2 \mathrm{MPa}$ and fall in the low modulus region of the stress-strain curve [Figure 3] which is similar to the value found for the modulus of skin in vivo [17]. Similar values of the modulus for cornea and sclera suggest that there is very little stress concentration at the cornea-scleral junction and that any stress concentration that occurs would probably reside at the scleral attachment laterally.It has been noted that blunt trauma causes scleral rupture by suddenly elevating intraocular pressure. Ruptures are most commonly observed at the insertions of the intraocular muscles or at the limbus, where the sclera is thinnest [22].

These measured values of modulus are similar to values reported in vivo for skin using vibrational OCT [16] [17]. The similarity between normal values of the moduli of skin, cornea and sclera suggest that the resting tension in these tissues is likely to be similar in vivo and this tension may act as a baseline for 
maintaining normal mechanotransduction. Increased or decreased tension (and moduli) may signal the up- or down regulation of mechanotransduction [23] that may ultimately lead to tissue fibrosis in skin, increased tension in glaucoma or tissue atrophy as observed in keratoconus.

The fact that the modulus calculated from different ECMs including skin, cartilage, cornea, sclera and dermis all are similar (Figure 5) suggests that fibrous collagen is the load bearing macromolecule in these tissues and other tissue components are in parallel with the collagen and do not limit tissue deformation or prevent premature mechanical failure [23]. In some vessel walls, other macromolecular networks such as smooth muscle are in series with the collagen network lowering the modulus of these tissues [24].

\section{Conclusion}

Our results indicate that the modulus of cornea and sclera can be measured non-invasively and non-destructively using vibrational OCT. This will assist clinicians to better understand the influence of biomechanics on the outcome of refractive surgery [25] and some common eye diseases such as glaucoma [26]-[32], myopia [33], and keratoconus [34] [35] [36]. It has been observed that the modulus of keratoconus corneas is lower than that of normal tissue [7] and that scleral stiffening occurs in glaucoma [37] associated with increased collagen alignment and birefringence [38]. Future studies with vibrational OCT will focus

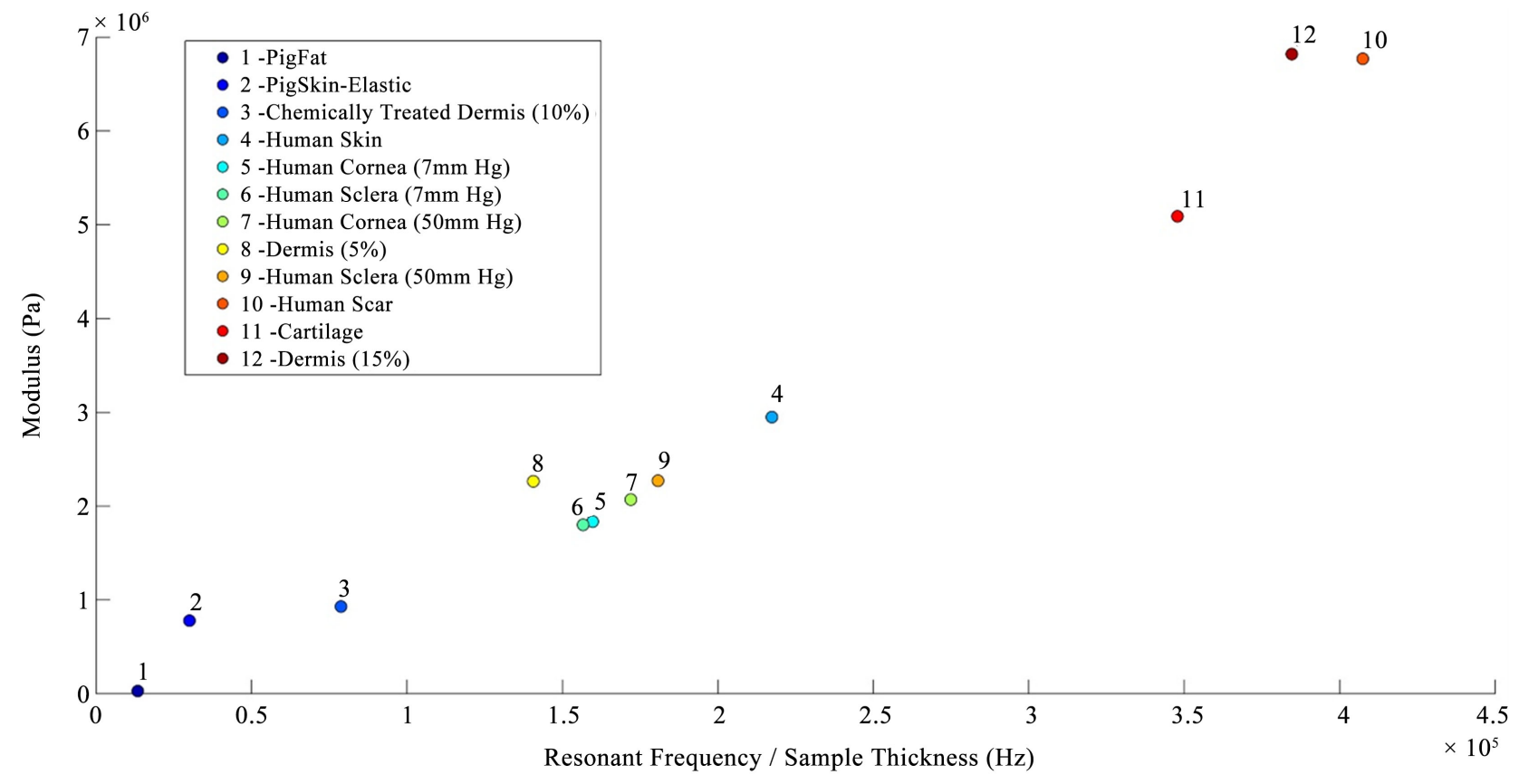

Figure 5. Plot of vibrational modulus versus the ratio of the resonant frequency per unit thickness for human decellularized dermis, human skin and scar, bovine cartilage, porcine fat and human cornea and sclera. The modulus for fat is about $0.03 \mathrm{MPa}$, elastic tissue 0.4 to $0.8 \mathrm{MPa}$, and collagen 2.0 to $7.0 \mathrm{MPa}$. Normal skin has a modulus of about $2 \mathrm{MPa}$ and scar of about $7 \mathrm{MPa}$. Normal cornea and sclera have moduli of about $2 \mathrm{MPa}$ at low and high intraocular pressures very similar to normal skin. Note that softening or stiffening of the ECMs found in ocular tissues would lead to lower (chemically treated dermis) or higher (scar) values of the moduli, respectively that would be measureable using vibrational OCT. 
on in vivo measurements of corneal and scleral biomechanics to study how collagen structure is altered in ocular diseases.

\section{References}

[1] Benedict, G. (1971) Theory of Transparency of the Eye. Applied Optics, 10, 459473. https://doi.org/10.1364/AO.10.000459

[2] Jue, B. and Maurice, D.M. (1986) The Mechanical Properties of the Rabbit and Human Cornea. Journal of Biomechanics, 19, 847-853.

https://doi.org/10.1016/0021-9290(86)90135-1

[3] Andreassan, T.T., Simonsen, A.H. and Oxlund, H. (1980) Biomechanical Properties of Keratoconus and Normal Corneas. Experimental Eye Research, 31, 435-441. https://doi.org/10.1016/S0014-4835(80)80027-3

[4] Roberts, C.J. (2014) Concepts and Misconceptions in Corneal Biomechanics. Journal of Cataract \& Refractive Surgery, 40, 862-869.

https://doi.org/10.1016/j.jcrs.2014.04.019

[5] Dupps Jr., W.J. and Wilson, S.E. (2006) Biomechanics and Wound Healing in the Cornea. Experimental Eye Research, 83, 709-720.

https://doi.org/10.1016/j.exer.2006.03.015

[6] Ruberti, J.W., Roy, A.S., Cynthia, J. and Roberts, C.J. (2011) Corneal Biomechanics and Biomaterials. Annual Review of Biomedical Engineering, 13, 269-295. https://doi.org/10.1146/annurev-bioeng-070909-105243

[7] Wollensak, G. and Iomdina, E. (2009) Biomechanical and Histological Changes after Corneal Crosslinking with and without Epithelial Debridement. Journal of Cataract \& Refractive Surgery, 35, 540-546. https://doi.org/10.1016/j.jcrs.2008.11.036

[8] Ambekara, R., Kimani, C., Toussaint Jr., B. and Johnson, A.W. (2011) The Effect of Keratoconus on the Structural, Mechanical, and Optical Properties of the Cornea. Journal of the Mechanical Behavior of Biomedical Materials, 4, 223-236. https://doi.org/10.1016/j.jmbbm.2010.09.014

[9] Nash, I.S., Peter, R., Green, P.R. and Foster, C.S. (1982) Comparison of Mechanical Properties of Keratoconus and Normal Corneas. Experimental Eye Research, 35, 413-423. https://doi.org/10.1016/0014-4835(82)90040-9

[10] Elsheikh, A. and Alhasso, D. (2009) Mechanical Anisotropy of Porcine Cornea and Correlation with Stromal Microstructure. Experimental Eye Research, 88, 1084-1091. https://doi.org/10.1016/j.exer.2009.01.010

[11] Elsheikh, A., Geraghty, B., Alhasso, D., Knappett, J., Campanelli, M. and Rama, P. (2010) Regional Variation in the Biomechanical Properties of the Human Sclera. Experimental Eye Research, 90, 624-633. https://doi.org/10.1016/j.exer.2010.02.010

[12] Silver, F.H. and Shah, R. (2016) Measurement of Mechanical Properties of Natural and Engineered Implants. Advances in Tissue Engineering and Regenerative Medicine, 1, 1-9.

[13] Yamada, H. and Evans, F.G. (1970) Strength of Biological Materials. University of Michigan.

[14] Fung, Y.C. (1993) Biomechanics: Mechanical Properties of Living Tissue. 2nd Edition, Springer. https://doi.org/10.1007/978-1-4757-2257-4

[15] Dunn, M.G. and Silver, F.H. (1983) Viscoelastic Behavior of Human Connective Tissues: Relative Contribution of Viscous and Elastic Components. Connective Tissue Research, 12, 59-70. https://doi.org/10.3109/03008208309005612

[16] Shah, R., Pierce, M.C. and Silver, F.H. (2017) A Method for Non-Destructive Me- 
chanical Testing of Tissues and Implants. Journal of Biomedical Materials Research Part $A$, 105, 15-22. https://doi.org/10.1002/jbm.a.35859

[17] Shah, R.G., DeVore, D. and Silver, F.H. (2018) Biomechanical Analysis of Decellularized Dermis and Skin: Initial in Vivo Observations Using Optical Cohesion Tomography and Vibrational Analysis. Journal of Biomedical Materials Research Part $A, 106,1421-1427$. https://doi.org/10.1002/jbm.a.36344

[18] Papagiannopoulos, G.A. and Hatzigeorgiou, G.D. (2011) On the Use of the Half-Power Bandwidth Method to Estimate Damping in Building Structures. Soil Dynamics and Earthquake Engineering, 31, 1075-1079. https://doi.org/10.1016/j.soildyn.2011.02.007

[19] Elsheikh, A., Alhasso, D. and Rama, P. (2008) Biomechanical Properties of Human and Porcine Corneas. Experimental Eye Research, 86, 783-790. https://doi.org/10.1016/j.exer.2008.02.006

[20] Shah, R.G. and Silver, F.H. (2017) Viscoelastic Behavior of Tissues and Implant materials: Estimation of the Elastic Modulus and Viscous Contribution Using Optical Coherence Tomography and Vibrational Analysis. Journal of Biomedical Technology and Research, 3, 105-109.

[21] Shah, R., DeVore, D. and Pierce, M.G. (2016) Morphomechanics of Dermis-A Method for Non-Destructive Testing of Collagenous Tissues. Skin Research and Technology, 23, 399-406. https://doi.org/10.1111/srt.12349

[22] Prabhat, K.P. and Sanaz, A.L. (2007) Ocular Emergencies. American Family Physician, 76, 829-836.

[23] Silver, F.H. and Silver, L.L. (2017) Gravity, Mechanotransduction and Healing. SM Journal of Biomedical Engineering, 3, 1023.

[24] Snowhill, P.B. and Silver, F.H. (2005) A Mechanical Model of Porcine Vascular Tissues-Part II: Stress-Strain and Mechanical Properties of Juvenile Porcine Blood Vessels. Cardiovascular Engineering, 5, 157-169.

https://doi.org/10.1007/s10558-005-9070-1

[25] Byun, Y., Kim, S., Lazo, M., Choi, M., Kang, M., Lee, J., Yoo, Y., Whang, W. and Joo, C. (2018) Astigmatic Correction by Intrastromal Astigmatic Keratotomy during Femtosecond Laser-Assisted Cataract Surgery: Factors in Outcomes. Journal of Cataract \& Refractive Surgery, 44, 202-208. https://doi.org/10.1016/j.jcrs.2017.11.018

[26] Sun, L., Shen, M., Wang, J., Fang, A., Xu, A., Fang, H. and Lu, F. (2009) Recovery of Corneal Hysteresis after Reduction of Intraocular Pressure in Chronic Primary Angle-Closure Glaucoma. American Journal of Ophthalmology, 6, 1061-1066. https://doi.org/10.1016/j.ajo.2009.01.008

[27] Pensyl, D., Sullivan-Mee, M., Torres-Monte, M., Halverson, K. and Qualls, C. (2012) Combining Corneal Hysteresis with Central Corneal Thickness and Intraocular Pressure for Glaucoma Risk Assessment. Eye (Lond), 10, 1349-1356. https://doi.org/10.1038/eye.2012.164

[28] Zhang, C., Tatham, A.J., Abe, R.Y., Diniz-Filho, A., Zangwill, L.M., Weinreb, R.N. and Medeiros, F.A. (2016) Corneal Hysteresis and Progressive Retinal Nerve Fiber Loss in Glaucoma. American Journal of Ophthalmology, 166, 29-36. https://doi.org/10.1016/j.ajo.2016.02.034

[29] Medeiros, F.A., Meira-Freitas, D., Lisboa, R., Kuang, T.M., Zangwill, L.M. and Weinreb, R.N. (2013) Corneal Hysteresis as a Risk Factor for Glaucoma Progression: A Prospective Longitudinal Study. Ophthalmology, 8, 1533-1540.

https://doi.org/10.1016/j.ophtha.2013.01.032 
[30] Park, J.H., Jun, R.M. and Choi, K.R. (2015) Significance of Corneal Biomechanical Properties in Patients with Progressive Normal-Tension Glaucoma. British Journal of Ophthalmology, 6, 746-751. https://doi.org/10.1136/bjophthalmol-2014-305962

[31] Mansouri, K., Leite, M.T., Weinreb, R.N., Tafreshi, A., Zangwill, L.M. and Medeiros, F.A. (2012) Association between Corneal Biomechanical Properties and Glaucoma Severity. American Journal of Ophthalmology, 3, 419-427. https://doi.org/10.1016/j.ajo.2011.08.022

[32] Vu, D.M., Silva, F.Q., Haseltine, S.J., Ehrlich, J.R. and Radcliffe, N.M. (2013) Relationship between Corneal Hysteresis and Optic Nerve Parameters Measured with Spectral Domain Optical Coherence Tomography. Graefe's Archive for Clinical and Experimental Ophthalmology, 7, 1777-1783. https://doi.org/10.1007/s00417-013-2311-x

[33] Chang, P.Y. and Chang, S.W. (2013) Corneal Biomechanics, Optic Disc Morphology, and Macular Ganglion Cell Complex in Myopia. Journal of Glaucoma, 22, 358-362. https://doi.org/10.1097/IJG.0b013e3182447a17

[34] Martinez-Abad, A. and Pinero, D.P. (2017) New Perspectives on the Detection and Progression of Keratoconus. Journal of Cataract \& Refractive Surgery, 43, 1213-1227. https://doi.org/10.1016/j.jcrs.2017.07.021

[35] Vinciguerra, R., Ambrosio, R., Elsheikh, A., Roberts, C.J., Lopes, B., Morenghi, E., Azzolini, C. and Vinciguerra, P. (2016) Detection of Keratoconus with a New Biomechanical Index. Journal of Refractive Surgery, 12, 803-810. https://doi.org/10.3928/1081597X-20160629-01

[36] Ambrosio, R., Lopes, B.T., Faria-Correia, F., Salomeo, M.Q., Buhren, J., Roberts, C.J., Elsheikh, A., Vinciguerra, R. and Vinciguerra, P. (2017) Integration of Scheimpflug-Based Corneal Topography and Biomechanical Assessments for Enhancing Ectasia Detection. Journal of Refractive Surgery, 7, 434-443. https://doi.org/10.3928/1081597X-20170426-02

[37] Huang, W., Fan, Q., Wang, W., Zhou, M., Laties, A.M. and Zhang, X. (2013) Collagen: A Potential Factor Involved in the Pathogenesis of Glaucoma. Medical Science Monitor Basic Research, 19, 237-240. https://doi.org/10.12659/MSMBR.889061

[38] Yamanari, M., Nagase, S., Fukuda, S., Ishii, K., Tanaka, R., Yasui, T., Oshika, T., Miura, M. and Yasuno, Y. (2014) Scleral Birefringence as Measured by Polarization-Sensitive Optical Coherence Tomography and Ocular Biometric Parameters of Human Eyes in Vivo. Optical Society of America, 5, 1391-1402.

https://doi.org/10.1364/BOE.5.001391 